\title{
Delaunay Properties of Digital Straight Segments
}

\author{
Tristan Roussillon ${ }^{1}$ and Jacques-Olivier Lachaud ${ }^{2}$ \\ 1 Université de Lyon, \\ Université Lyon 2, LIRIS, UMR5205, F-69676, France \\ tristan.roussillon@liris.cnrs.fr \\ 2 LAMA, UMR CNRS 5127 \\ Université de Savoie, Le Bourget-du-Lac, 73376, France \\ jacques-olivier.lachaud@univ-savoie.fr
}

\begin{abstract}
We present new results concerning the Delaunay triangulation of the set of points of pieces of digital straight lines. More precisely, we show how the triangulation topology follows the arithmetic decomposition of the line slope as well as its combinatorial decomposition (splitting formula). A byproduct is a linear time algorithm for computing the Delaunay triangulation and the Voronoi diagram of such sets.
\end{abstract}

\section{Introduction}

Let $S$ be a set of $n$ points in $\mathbb{R}^{2}$. Its convex hull is the intersection of every half-planes containing $S$. A triangulation of $S$ is a simplicial decomposition of the convex hull of $S$ where the vertices of the triangular facets are elements of $S$. The Delaunay triangulation of $S$ is a triangulation such that each facet satisfies the Delaunay condition: the circumcircle of the facet contains no point from $S$ in its interior. Such a triangulation exists for every point set in $\mathbb{R}^{2}$ (and more generally in arbitrary dimension), and it is the dual of the Voronoi diagram. It plays a very important role in computational geometry and meshing. A lot of algorithms exist to compute it, and have optimal time complexity $O(n \log n)[7$.

The Delaunay triangulation of a set of Euclidean points has been deeply studied in computational geometry. The special case of a set of digital points (points in the digital plane $\mathbb{Z}^{2}$ ) is generally not addressed as is, but more as a binary image. In this context, euclidean distance transforms provide a way of computing digital Voronoi diagram i.e. each pixel is labeled according to its closest site, but does not give the discrete Voronoi diagram, i.e. the set of vertices, edges of the diagram and its topology, and thus do not give the Delaunay triangulation either.

In this paper we study precisely the properties of the Delaunay triangulation of specific subsets of the digital plane $\mathbb{Z}^{2}$, which are digital equivalents of straight lines and segments. The standard line $D(a, b, \mu)$ of slope $\frac{a}{b}$ and intercept $\mu$ is the set of point $(x, y) \in \mathbb{Z}^{2}$ verifying $\mu \leq a x-b y<\mu+|a|+|b|$ with $a$, $b, \mu$ integer and $\operatorname{gcd}(a, b)=1[9]$. The points verifying $a x-b y=\mu$ (resp. 
$a x-b y=\mu+|a|+|b|-1$ ) are called the upper (resp. lower) leaning points. The connected part of a standard line $D(a, b, \mu)$ between two consecutive upper leaning points $U$ and $U^{\prime}=U+(b, a)$ is called a pattern. It is a 4-connected path joining $U$ and $U^{\prime}$, conveniently denoted by $U U^{\prime}$. Its staircase representation is the polygonal line joining the points of the pattern in the same order as the 4-connected path does. The chain code of a pattern is a Christoffel word. The slope of a pattern is the slope of its vector $\overrightarrow{U U^{\prime}}=(b, a)$, i.e. $\frac{a}{b}$. These standard definitions are illustrated on Fig. 1a. Note that any digital straight segment has a unique decomposition into patterns, which makes patterns very important for analyzing digital shapes.

We show in Section 2 that the Delaunay triangulation of a pattern has very specific properties. They are related to the specific geometry of points within a pattern. From an equivalent arithmetic point of view, they are related to the continued fraction of the pattern slope or, from a combinatorial point of view, to the splitting formula (e.g. see 5]). With these properties, we deduce in Section 3 a linear time algorithm to compute the Delaunay triangulation and the Voronoi diagram of such sets of points. The presented results may have several interesting applications in computational geometry and digital shape analysis which are discussed in Section 4.

\section{Delaunay Properties of Patterns}

Before stating precisely the main theorem, we introduce briefly some further notions. From now on, we restrict our study to the first quadrant. Let $U U^{\prime}$ be the pattern of the standard line $D(a, b, 0)$ between $U=(0,0)$ and $U^{\prime}=(b, a)$ with $a>0$ and $b>0$.

\subsection{Triangulation of a Pattern}

Let us denote by $\mathcal{H}\left(U U^{\prime}\right)$ the convex hull of $U U^{\prime}$. The staircase representation of $U U^{\prime}$ is by definition below the straight segment $\left[U U^{\prime}\right]$. Therefore, $\mathcal{H}\left(U U^{\prime}\right)$ can be divided into two parts lying on both sides of its staircase representation: the upper part $\mathcal{H}^{+}\left(U U^{\prime}\right)$, whose boundary contains $\left[U U^{\prime}\right]$, in light gray fig [1.b, and the lower one $\mathcal{H}^{-}\left(U U^{\prime}\right)$, in dark gray fig [1, b.

Let us denote by $\mathcal{T}\left(U U^{\prime}\right)$ the Delaunay triangulation of $U U^{\prime}$. We will see that it can similarly be divided into two parts $\mathcal{T}^{+}\left(U U^{\prime}\right)$ and $\mathcal{T}^{-}\left(U U^{\prime}\right)$, separated by the staircase representation. Fig. 1, b shows the Delaunay triangulation of a pattern of slope $\frac{4}{7}$.

We introduce here three equivalent definitions of the main facet of a pattern (see Fig. 2).

Definition 1 (main facet). The main facet of a pattern $U U^{\prime}$ is the triangle $U B U^{\prime}$, where $B$ has the following equivalent definitions:

(geometric) the digital point $B$ within $U U^{\prime} \backslash\left\{U, U^{\prime}\right\}$ that has the shortest orthogonal distance to the segment $\left[U U^{\prime}\right]$; 


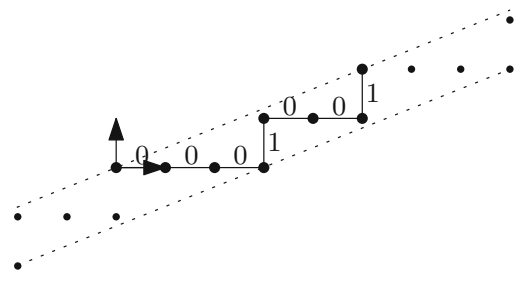

(a)

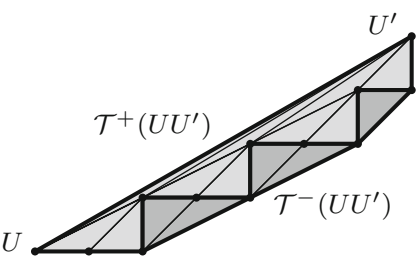

(b)

Fig. 1. (a) The points of $D(2,5,0)$ are depicted with black disks. The two dotted straight lines pass through the upper and lower leaning points. A pattern is highlighted with bigger disks. The letters of its chain code are indicated near its staircase representation, which is depicted with a solid line. (b) The Delaunay triangulation of the pattern $U U^{\prime}$ is divided into the upper part $\mathcal{T}^{+}\left(U U^{\prime}\right)$, which contains the facets lying in $\mathcal{H}^{+}\left(U U^{\prime}\right)$, in light gray, and the lower part $\mathcal{T}^{-}\left(U U^{\prime}\right)$, which contains the facets lying in $\mathcal{H}^{-}\left(U U^{\prime}\right)$, in dark gray.

(arithmetic) the lower Bezout point $B$ to the vector $\overrightarrow{U U^{\prime}}$;

(combinatorial) the separation point $B$ of the splitting formula (see [10], chap. 4).

These characterizations are shown equivalent later in the paper.

Definition 2 (facets of a pattern). The facets of the pattern $U U^{\prime}$, denoted by $\mathcal{F}\left(U U^{\prime}\right)$, is defined recursively as the union of the main facet $U B U^{\prime}$ of $U U^{\prime}$ and the facets of the pattern $U B$ if $a>1$ and the facets of the pattern $B U^{\prime}$ if $b>1$.

Note that this recursive definition is consistent because (i) $U B$ and $B U^{\prime}$ are both patterns (see Proposition 1 1ater), (ii) $a+b$ strictly decreases so that both $a$ and $b$ reach 1 and the recursion terminates.

\subsection{Main Result}

We can now state precisely our main result.

Theorem 1. The facets of the pattern $U U^{\prime}$ is a triangulation of $\mathcal{H}^{+}\left(U U^{\prime}\right)$. Furthermore, each facet satisfies the Delaunay property, i.e. the circumcircle of each triangular facet of $\mathcal{F}\left(U U^{\prime}\right)$ contains none of the points of the pattern $U U^{\prime}$ in its interior.

In other words, the facets of the pattern $U U^{\prime}$ is exactly $\mathcal{T}^{+}\left(U U^{\prime}\right)$.

We prove this theorem in the following subsections. We prove first that the facets form a triangulation (Theorem 2), then that each of them has the Delaunay property (Theorem 3). 


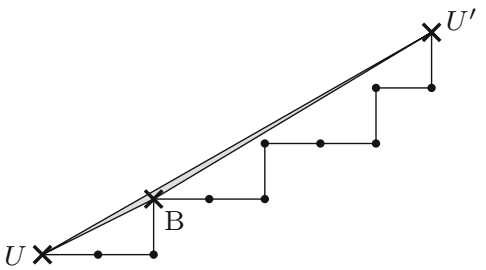

(a)

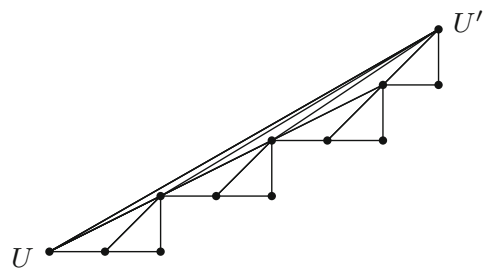

(b)

Fig. 2. (a) The gray area, bounded by the triangle $U B U^{\prime}$, is the main facet of $U U^{\prime}$. Note that $B=b^{-}\left(U U^{\prime}\right)$ belongs to $U U^{\prime}$ and that $U B$ and $B U^{\prime}$ are both patterns. (b) The facets of $U U^{\prime}$ are defined by induction over the patterns included in $U U^{\prime}$.

\subsection{The Facets of a Pattern Form a Triangulation}

We recall that the remainder with respect to the standard line of slope $\frac{a}{b}$ is a function $r_{a, b}: \mathbb{Z}^{2} \rightarrow \mathbb{Z}$ such that $r_{a, b}(x, y)=a x-b y$. In the sequel, $r_{a, b}(\cdot)$ is simplified into $r(\cdot)$ when the remainder refers to the standard line $D(a, b, 0)$ containing $U U^{\prime}$.

Definition 3 (support of a pattern). The support of the pattern $U U^{\prime}$ is the set of lattice points $S\left(U U^{\prime}\right)$ lying in a strip bounded by two straight lines orthogonal to the straight line $\left(U U^{\prime}\right)$ and passing through $U$ and $U^{\prime}$, i.e. $S\left(U U^{\prime}\right)=$ $\left\{(x, y) \in \mathbb{Z}^{2} \mid 0 \leq b x+a y \leq a^{2}+b^{2}-1\right\}$.

Given an integer $k$, let the straight line $\mathcal{L}_{k}$ goes through the lattice points $X$ of remainder $r(X)=k$. The lattice points of $\mathcal{L}_{k}$ are regularly spaced of a distance equal to $\sqrt{a^{2}+b^{2}}$. Since the points of $S\left(U U^{\prime}\right)$ lie in a strip of width strictly less than $\sqrt{a^{2}+b^{2}}, \mathcal{L}_{k}$ contains only one point of $S\left(U U^{\prime}\right)$ for each $k$. Thus, we may define (see Fig. 2a):

Definition 4 (Bezout point). The lower Bezout point of the pattern $U U^{\prime}$, denoted by $b^{-}\left(U U^{\prime}\right)$ is the unique point of $S\left(U U^{\prime}\right)$ whose remainder equals to 1 .

Note that $b^{-}\left(U U^{\prime}\right)$, whose remainder equals to 1 , belongs to the pattern $U U^{\prime}$ contained in $D(a, b, 0)$ if and only if $|a|+|b| \geq 2$, i.e. $a \neq 0$ and $b \neq 0$. Considering that the orthogonal distance of a lattice point $P$ with respect to the line $U U^{\prime}$ is $\left|r(P) / \sqrt{a^{2}+b^{2}}\right|$, it is clear that the Bezout point to $U U^{\prime}$ is the point of $U U^{\prime} \backslash\left\{U, U^{\prime}\right\}$ closest to the segment $\left[U U^{\prime}\right]$, which proves the equivalence between the geometric and arithmetic definition of the main facet (Definition 1).

Note that the main facet of a pattern never contains any lattice points:

Proposition 1. Let $X$ be a lattice point defined with a positive integer $k$ such that $r(X)=k$. The triangle $U X U^{\prime}$ contains in its interior one or more lattice points if and only if $k>1$.

Proposition 1, which is directly proved with Pick formula, explains why, if $b^{-}\left(U U^{\prime}\right)$ belongs to $U U^{\prime}$, the 4-path joining $U$ to $b^{-}\left(U U^{\prime}\right)$ as well as the one 
joining $b^{-}\left(U U^{\prime}\right)$ to $U^{\prime}$, are both patterns. Therefore the inductive definition of the facets of a pattern (Definition 2) makes sens. The number of facets of a pattern is finite because $\mathcal{F}\left(U U^{\prime}\right)$ coincides with $\mathcal{H}^{+}\left(U U^{\prime}\right)$ (compare Fig. 2, b and Fig. 1].b), as shown below:

Theorem 2. The facets of a pattern $U U^{\prime}$ is a triangulation of $\mathcal{H}^{+}\left(U U^{\prime}\right)$.

Proof. We shall prove this assertion by structural induction on the recursive definition of the facets of a pattern.

(i) Base case: Let us take a pattern with $a=1$ and $b=1$. The facets of $U U^{\prime}$ are then reduced to the main facet $U B U^{\prime}$ which is the triangle $(0,0),(0,1),(1,1)$. It is equal to its convex hull $\mathcal{H}^{+}\left(U U^{\prime}\right)$ and is thus its triangulation.

(ii) Inductive step: Given a pattern $U U^{\prime}$ with $a>1$ or $b>1$, let us assume that the facets of $U B$ is a triangulation of $\mathcal{H}^{+}(U B)$ and the facets of $U B^{\prime}$ is a triangulation of $\mathcal{H}^{+}\left(B U^{\prime}\right)$. We shall prove that the facets of $U U^{\prime}$ is a triangulation of $\mathcal{H}^{+}\left(U U^{\prime}\right)$.

First of all, since $\mathcal{H}^{+}(U B)$ is below $[U B]$ and $\mathcal{H}^{+}\left(B U^{\prime}\right)$ is below $\left[B U^{\prime}\right]$, both have thus an empty intersection with the interior of the triangle $U B U^{\prime}$ (the main facet of $\left.U U^{\prime}\right)$. Furthermore, $\mathcal{H}^{+}\left(U U^{\prime}\right)$ forms a simple polygon (Euler characteristics is 2) since it is the union of three simple polygons, with one more edge $\left(\left[U U^{\prime}\right]\right)$ and one more face $\left(U B U^{\prime}\right)$ than the union of $\mathcal{H}^{+}(U B)$ and $\mathcal{H}^{+}\left(B U^{\prime}\right)$. Lastly, the boundary edges of the facets of $U U^{\prime}$ are exactly the boundary edges of $\mathcal{H}^{+}\left(U U^{\prime}\right)$ : the main facet has its edge $\left[U U^{\prime}\right]$ that is the upper edge of $\mathcal{H}^{+}\left(U U^{\prime}\right)$, while the lower edges of $\mathcal{H}^{+}(U B)$ and $\mathcal{H}^{+}\left(B U^{\prime}\right)$ define the staircase representation of $U U^{\prime}$, which is by definition the lower edges of $\mathcal{H}^{+}\left(U U^{\prime}\right)$.

The facets of $U U^{\prime}$ thus define a triangulation of a simple polygon whose (only) boundary is the same as the boundary of $\mathcal{H}^{+}\left(U U^{\prime}\right)$. It is thus a triangulation of $\mathcal{H}^{+}\left(U U^{\prime}\right)$.

\subsection{Delaunay Condition for Each Facet}

It remains to prove that each facet of $\mathcal{F}\left(U U^{\prime}\right)$ satisfies the Delaunay condition. In the whole subsection, we denote by $B$ the lower Bezout point of the pattern $U U^{\prime}(a>0$ and $b>0)$. Let us begin by a lemma that completes Proposition 1,

Lemma 1. For all $P \in S\left(U U^{\prime}\right)$ with $r(P)>1$, the triangle $U P U^{\prime}$ contains $B$.

Lemma 1 and its proof are illustrated in Fig. 3. a.

Proof. For all $P \in S\left(U U^{\prime}\right)$ such that $r(P)>1$, we prove below that the slope of $\overrightarrow{U P}$ is smaller than the one of $\overrightarrow{U B}$ and similarly that the slope of $\overrightarrow{P U^{\prime}}$ is greater than the one of $\overrightarrow{B U^{\prime}}$, which prove that the triangle $U P U^{\prime}$ contains the triangle $U B U^{\prime}$ and thus $B$.

Given a positive integer $k$, let $B_{k}=k \overrightarrow{U B}$. We have $B=B_{1} \in S\left(U U^{\prime}\right)$ and $r\left(B_{k}\right)=k$. Let $k_{0}$ be the greatest $k>1$ such that $B_{k-1} \in S\left(U U^{\prime}\right)\left(k_{0}=3\right.$ in Fig. 3. a). The lattice point $B_{k_{0}}$ is not in $S\left(U U^{\prime}\right)$ and $k_{0} \geq 2$. Hereafter, we independently deal with the points of $S\left(U U^{\prime}\right)$ of remainder ranging from 2 to $k_{0}-1$ (i) and greater than or equal to $k_{0}$ (ii). 
(i) For the $k_{0}-2$ points $P$ of $S\left(U U^{\prime}\right)$ such that $1<r(P)<k_{0}, \overrightarrow{U P}$ has the same slope as $\overrightarrow{U B}$.

(ii) For any point $X$, the tangent of the angle $\angle X U U^{\prime}$ is equal to the ratio between the determinant of $\overrightarrow{U X}, \overrightarrow{U U^{\prime}}$ and the scalar product of $\overrightarrow{U X}, \overrightarrow{U U^{\prime}}$.

The scalar product with $\overrightarrow{U U^{\prime}}$ defines the length of the orthogonal projection on the line $\left(U U^{\prime}\right)$. It is obvious that the length of the projection of a point outside the orthogonal strip $S\left(U U^{\prime}\right)$ is greater than the length of the projection of any point $P$ within this strip, thus $\overrightarrow{U B_{k_{0}}} \cdot \overrightarrow{U U^{\prime}}>\overrightarrow{U P} \cdot \overrightarrow{U U^{\prime}}$.

On the other hand, the determinant between $\overrightarrow{U B_{k_{0}}}$ and $\overrightarrow{U U^{\prime}}$, which is equal to $r\left(B_{k_{0}}\right)=k_{0}$, is smaller than or equal to the determinant between $\overrightarrow{U P}$ and $\overrightarrow{U U^{\prime}}$ for all $P \in S\left(U U^{\prime}\right)$ such that $r(P) \geq k_{0}$.

$$
\tan \left(\angle B U U^{\prime}\right)=\tan \left(\angle B_{k_{0}} U U^{\prime}\right)=\frac{\operatorname{det}\left(\overrightarrow{U B_{k_{0}}}, \overrightarrow{U U^{\prime}}\right)}{\overrightarrow{U B_{k_{0}}} \cdot \overrightarrow{U U^{\prime}}}<\frac{\operatorname{det}\left(\overrightarrow{U P}, \overrightarrow{U U^{\prime}}\right)}{\overrightarrow{U P} \cdot \overrightarrow{U U^{\prime}}}=\tan \left(\angle P U U^{\prime}\right)
$$

As a consequence, the slope of $\overrightarrow{U P}$ is smaller than the one of $\overrightarrow{U B}$ for all $P \in$ $S\left(U U^{\prime}\right)$ such that $r(P)>1$. We can similarly show that the slope of $\overrightarrow{P U^{\prime}}$ is greater than the one of $\overrightarrow{B U^{\prime}}$, which concludes the proof.

Lemma 1 is used in Lemma 2 and Lemma 4.

Lemma 2. Let $\mathcal{D}$ be a disk whose boundary passes through $U$ and $U^{\prime}$ and whose center is located above $\left(U U^{\prime}\right)$. Let $\partial \mathcal{D}$ be its boundary. $\mathcal{D} \backslash \partial \mathcal{D}$ contains a lattice point below or on $\left(U U^{\prime}\right)$ if and only if it contains (at least) B (Fig. 3. b).

Proof. Given a positive integer $k$, let $\mathcal{L}_{k}$ be the straight line passing through the points of remainder $k$. Let $S_{\mathcal{D}}$ be the set of lattice points of strictly positive remainder contained in $\mathcal{D}$. Since the center of $\mathcal{D}$ is located above $\left(U U^{\prime}\right)$, the length of the segment $\mathcal{L}_{k} \cap \mathcal{D}$ is smaller than $\sqrt{a^{2}+b^{2}}$ for all $k>0$. Thus $S_{\mathcal{D}} \subset S\left(U U^{\prime}\right)$ (Fig. 3, b).

Due to Lemma 1] (Fig. 3. a), $\angle U P U^{\prime} \leq \angle U B U^{\prime}$ for all $P \in S\left(U U^{\prime}\right)$ such that $r(P) \geq 1$, which concludes for all lattice points strictly below $\left(U U^{\prime}\right)$.

Since $\left[U U^{\prime}\right]$ does not contain any lattice points, except $U$ and $U^{\prime}, \mathcal{D} \backslash \partial \mathcal{D}$ does not contain any lattice point on $\left(U U^{\prime}\right)$, which concludes.

Let us now introduce the following key definition:

Definition 5 (background of a pattern). The background of the pattern $U U^{\prime}$ is the set of lattice points located below or on the straight line $(U B)$, or below or on the straight line $\left(B U^{\prime}\right)$.

Definition 5, illustrated in Fig. 4, is used in Lemma 3 and Lemma 4.

Lemma 3. Let $\mathcal{D}$ be a disk whose boundary $\partial \mathcal{D}$ is the circumcircle of $U B U^{\prime}$. The disk $\mathcal{D}$ contains none of the background points of $U U^{\prime}$, except $U, U^{\prime}, B$, which are all located on $\partial \mathcal{D}$ (Fig. 4. a). 


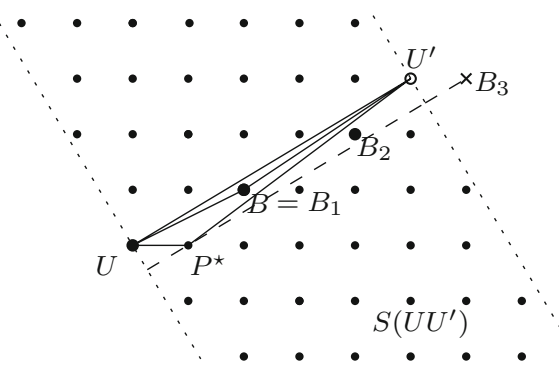

(a)

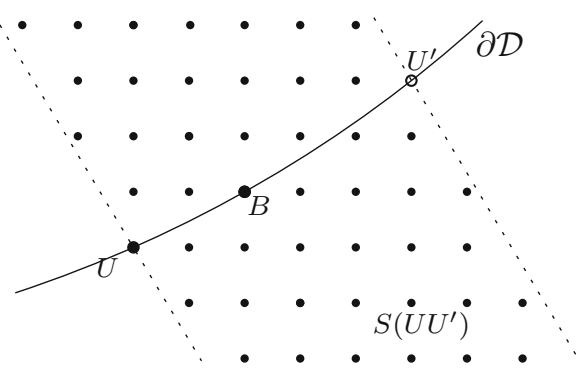

(b)

Fig. 3. (a) Illustration of Lemma1 The triangle $U P^{\star} U^{\prime}$ contains $B$ because $P^{\star}$ belongs to $S\left(U U^{\prime}\right)$ (depicted by the set of black disks) and $r\left(P^{\star}\right)>1$. The points $B_{k}=k \overrightarrow{U B}$ (indicated for $k=1,2,3)$ are used in the proof of Lemma 1 to show that the slope of $\overrightarrow{U P}$ is smaller than the one of $\overrightarrow{U B}$ for all $P \in S\left(U U^{\prime}\right)$ such that $r(P)>1$. (b) Illustration of Lemma $2 \mathcal{D}$ is a disk whose boundary $\partial \mathcal{D}$ pass through $U, U^{\prime}$ and $B$. The interior $\mathcal{D} \backslash \partial \mathcal{D}$ does not contain any lattice points located below or on the straight line $\left(U U^{\prime}\right)$ due to Lemma 1 .

Proof. Let $B_{l}$ (resp. $B_{r}$ ) be the lower Bezout point of $U B$ (resp. $B U^{\prime}$ ).

The proof is based on the two following arguments:

1. $\mathcal{D}$ contains neither $B_{l}$ nor $B_{r}$.

2. $\mathcal{D}$ contains neither the set of lattice points located below or on $(U B)$, nor the set of lattice points located below or on $\left(B U^{\prime}\right)$.

Assuming 1) is true, argument 2) follows from Lemma 2 applied once for pattern $U B$ and once for pattern $B U^{\prime}$, which in turn concludes the proof due to the definition of background (Definition [5).

Let us now prove argument 1). Moreover let us focus on $B_{l}$ because the proof about $B_{r}$ is similar. According again to Lemma 2, it is enough to show that the remainder of $B_{l}$ is strictly positive so as to show it is below $\left(U U^{\prime}\right)$.

If $a=1, B=(1,0), B_{l}=(0,-1)$ and $r\left(B_{l}\right)=b$. Thus, $r\left(B_{l}\right) \geq 1$.

If $a>1, B_{l}$ belongs to the pattern $U B$, which belongs to the pattern $U U^{\prime}$. By definition, all the points $X \in U U^{\prime} \backslash U^{\prime}$ belong to $S\left(U U^{\prime}\right)$ and have a remainder $r(X)$ ranging from 0 to $|a|+|b|-1>1$. Moreover, given an integer $k$, there is only one point $X \in S\left(U U^{\prime}\right)$ such that $r(X)=k$. Since $B_{l} \neq B$, we conclude that $r\left(B_{l}\right)>1$.

Lemma 4. The background of $U U^{\prime}$ is contained in the background of $U B$ if $a>1$ and in the one of $B U^{\prime}$ if $b>1$ (Fig. 4. $b$ ).

Proof. Let us assume that $a>1$, the case where $b>1$ being symmetric.

Let $B_{l}$ be the lower Bezout point of $U B$. As in the proof of Lemma 3, it can be shown that $r\left(B_{l}\right)>1$. Due to Lemma 1, the triangle $U B_{l} U^{\prime}$ contains the triangle $U B U^{\prime}$. 
As a consequence, the slope of $\overrightarrow{B_{l} B}$ is greater than the one of $\overrightarrow{B U^{\prime}}$. Since the triangle $U B_{l} B$ does not contain any lattice points due to Proposition 1 , the set of lattice points whose $\mathrm{x}$-coordinate is greater than $B_{l}$ and lying below or on the straight line $\left(B_{l} B\right)$ contains the set of lattice points whose $\mathrm{x}$-coordinate is greater than $B_{l}$ and lying below or on the straight line $\left(B U^{\prime}\right)$.

Similarly the slope of $\overrightarrow{U B_{l}}$ is smaller than the one of $\overrightarrow{U B}$. Since the triangle $U B_{l} B$ does not contain any lattice points due to Proposition 1, the set of lattice points whose x-coordinate is smaller than $B_{l}$ and lying below or on the straight line $\left(U B_{l}\right)$ contains the set of lattice points whose $\mathrm{x}$-coordinate is smaller than $B_{l}$ and lying below or on the straight line $(U B)$.

Due to the definition of background (Definition 5), we can conclude that the background of $U B$ contains the background of $U U^{\prime}$ (like in Fig. 4. b), which concludes the proof.

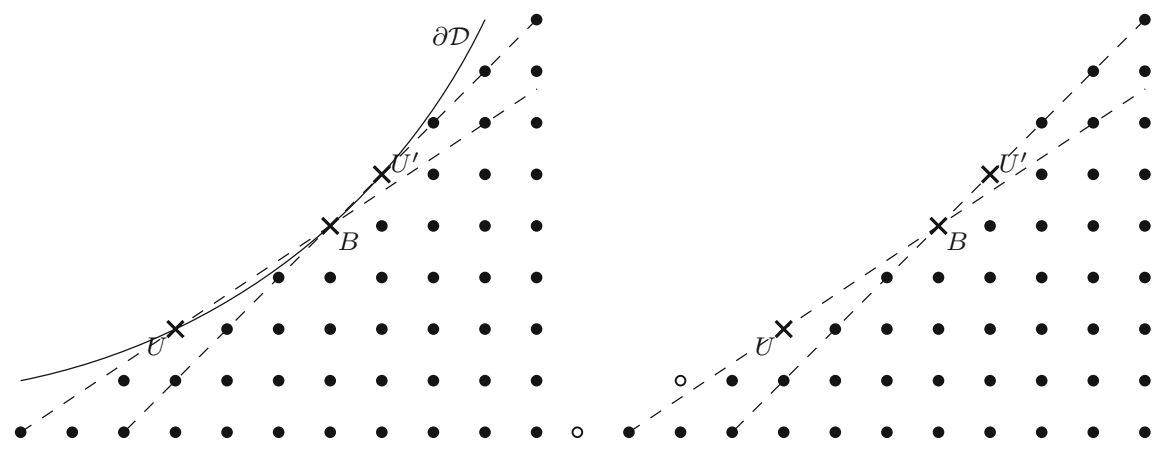

(a)

(b)

Fig. 4. (a) Illustration of Lemma 3 The set of lattice points located below $U B$ or $B U^{\prime}$, depicted with black disks, is the background of $U U^{\prime}$. The disk $\mathcal{D}$ passing through $U$, $B, U^{\prime}$ contains none of the points of the background $U U^{\prime}$. (b) Illustration of Lemma 4 The background of $U U^{\prime}$ is contained in the background of $U B$, which has extra points, the two visible ones are depicted with white disks.

We can now prove the Delaunay condition for each facet of $\mathcal{F}\left(U U^{\prime}\right)$ :

Theorem 3. The circumcircle of each triangular facet of $\mathcal{F}\left(U U^{\prime}\right)$ contains none of the background points of $U U^{\prime}$ in its interior.

Proof. We shall prove this assertion by structural induction on the recursive definition of the facets of a pattern.

(i) Base case: Let us take a pattern with $a=1$ and $b=1$. The facets of $U U^{\prime}$ are then reduced to the main facet $U B U^{\prime}$, which is the triangle $(0,0),(0,1),(1,1)$. The property is trivial by definition of the circumcircle.

(ii) Inductive step: Let $U B$ and $B U^{\prime}$ be two patterns such that $B$ is the lower Bezout point of the pattern $U U^{\prime}$ with $a>1$ or $b>1$. Let us assume that Theorem 3 is true for $U B$ and $B U^{\prime}$. We shall prove that Theorem 3 is true for $U U^{\prime}$. 
Lemma 3 concludes for the main facet $U B U^{\prime}$. We observe now the facets of $U U^{\prime}$ that are also facets of $U B$ or $B U^{\prime}$. Since $a>1$ or $b>1$, the background of $U B$ or $B U^{\prime}$ contains the one of $U U^{\prime}$ due to Lemma 4 . Since the circumcircle of each triangular facet of $\mathcal{F}(U B)$ (resp. $\mathcal{F}\left(B U^{\prime}\right)$ ) contains none of the background points of $U B$ (resp. $B U^{\prime}$ ) in its interior due to the induction hypothesis, it does not contain any background point of $U U^{\prime}$ either. We can thus conclude.

Since $U U^{\prime}$ is contained in its background $\left(U B U^{\prime}\right.$ does not contain any lattice point due to Proposition 11), Theorems 2 and 3 clearly imply Theorem [1. The facets of the pattern $U U^{\prime}$ coincides with the upper part of the Delaunay triangulation of $U U^{\prime}$, i.e. $\mathcal{F}\left(U U^{\prime}\right)=\mathcal{T}^{+}\left(U U^{\prime}\right)$. It remains only to show that the combinatorial characterization of the main facet (Definition 1) is the same as either the arithmetic one or geometric one. This is done in the next section.

\section{Applications}

In this section, we explain how to efficiently compute the Delaunay triangulation or the Voronoi diagram of the pattern $U U^{\prime}$ from the continued fraction expansion of its slope.

\subsection{Continued Fraction}

Let $\left[q_{0} ; \ldots, q_{i}, \ldots, q_{n}\right]$ (with $\left.q_{n}>1\right)$ be the quotients and $\left(b_{0}, a_{0}\right), \ldots,\left(b_{i}, a_{i}\right)$, $\ldots,\left(b_{n}, a_{n}\right)$ be the convergent vectors of the continued fraction expansion of $\frac{a}{b}$. Note that $\left(b_{n}, a_{n}\right)$ is the closest approximation of $(b, a)$ (without being equal to $(b, a))$, because $q_{n}$ is assumed to be greater than 1 . Let $\left(b_{n+1}, a_{n+1}\right)$ be equal by convention to $(b, a)$. The sequence of convergents verifies:

$$
\forall i \in 1, \ldots, n,\left(b_{i+1}, a_{i+1}\right)=\left(b_{i-1}, a_{i-1}\right)+q_{i}\left(b_{i}, a_{i}\right)
$$

Moreover, for all $i \in 1, \ldots, n$, the determinant of $\left(b_{i}, a_{i}\right)$ and $\left(b_{i+1}, a_{i+1}\right)$ is equal to 1 if $i$ is odd and -1 if $i$ is even.

For instance, the continued fraction representation of $\frac{3}{5}$ is $[0 ; 1,1,2]$ and the sequence of its convergent vectors is $(0,1),(1,0),(1,1),(2,1)$. Note that $(5,3)=$ $(1,1)+2(2,1)$ (and $(2,1)=(1,0)+1(1,1)$, etc.) as expected from eq. 1. In addition, $5.1-3.2=-1$ (and $2.1-1.1=1$, etc.). This suggests that the lower Bezout point $B$ is available from the quotients and the convergent vectors of the continued fraction expansion of the slope $\frac{a}{b}$ of $U U^{\prime}$ and indeed:

$$
\overrightarrow{U U^{\prime}}=\overrightarrow{U B}+\overrightarrow{B U^{\prime}}=\left(b_{n}, a_{n}\right)+\left(\left(b_{n-1}, a_{n-1}\right)+\left(q_{n}-1\right)\left(b_{n}, a_{n}\right)\right)
$$

Eq. 2 is a formulation in terms of convergent vectors of the splitting formula, originally expressed in terms of quotients [10, Theorem 4.1]. The combinatorial characterization of the main facet of Definition 1] is therefore the same as the arithmetic one. 
Note that $\left(b_{n}, a_{n}\right)=\overrightarrow{U B}$ if $n$ is odd, but $\left(b_{n}, a_{n}\right)=\overrightarrow{B U^{\prime}}$ if $n$ is even. For instance, if $\overrightarrow{U U^{\prime}}=(5,3)$, the last convergent vector is $\overrightarrow{U B}=(2,1)$ and $\overrightarrow{B U^{\prime}}=$ $(1,1)+(2-1) \cdot(2,1)=(3,2)$.

Furthermore, the description of the slopes of $U B$ and $B U^{\prime}$ can be easily deduced from the one of $U U^{\prime}$ : the quotients and convergent vectors associated to the slope of $U B$ is $\left[q_{0} ; \ldots, q_{n-1}\right]$ and $\left(b_{0}, a_{0}\right), \ldots,\left(b_{n-1}, a_{n-1}\right)$ if $n$ is odd, $\left[q_{0} ; \ldots, q_{n}-1\right]$ and $\left(b_{0}, a_{0}\right), \ldots,\left(b_{n}, a_{n}\right)$ if $n$ is even, whereas the quotients and convergent vectors associated to the slope of $B U^{\prime}$ is $\left[q_{0} ; \ldots, q_{n}-1\right]$ and $\left(b_{0}, a_{0}\right), \ldots,\left(b_{n}, a_{n}\right)$ if $n$ is odd, $\left[q_{0} ; \ldots, q_{n-1}\right]$ and $\left(b_{0}, a_{0}\right), \ldots,\left(b_{n-1}, a_{n-1}\right)$ if $n$ is even.

\subsection{Computation of the Delaunay Triangulation}

The computation of the Delaunay triangulation of $\mathcal{T}^{-}\left(U U^{\prime}\right)=\mathcal{F}\left(U U^{\prime}\right)$ is made by a simple recursive algorithm, which may be coarsely describe as follows: find $B=b^{-}\left(U U^{\prime}\right)$, the lower Bezout point of $U U^{\prime}$, using the extended Euclidean algorithm, add the triangular facet $U B U^{\prime}$ to $\mathcal{F}\left(U U^{\prime}\right)$ and split $U U^{\prime}$ into $U B$ and $B U^{\prime}$ in order to recursively apply the procedure on sub-patterns. The algorithm stops when the sub-patterns are as small as two consecutive points of $U U^{\prime}$.

Though, it is enough to run only once the extended Euclidean algorithm because the sequence of convergent vectors of any sub-patterns is computed in constant time from its parent pattern as said in the previous subsection.

To sum up, the extended Euclidean algorithm is only run once at the beginning in $\mathcal{O}\left(\log ^{2}(\max (|a|,|b|))\right)$. Then, each step requires $\mathcal{O}(1)$ time to draw a new triangular facet. Since there are exactly $|a|+|b|+1$ points and $|a|+|b|-1$ facets, the whole algorithm is in $\mathcal{O}(|a|+|b|)$, whereas any classic algorithm coming from computational geometry is in $\mathcal{O}((|a|+|b|) \log (|a|+|b|))$ in the RAM model.

To end, note that $\mathcal{T}^{-}\left(U U^{\prime}\right)$, the lower part of the Delaunay triangulation of $U U^{\prime}$, is the union of the facets of all the reversed patterns $L L^{\prime}$ such that the straight segment $\left[L L^{\prime}\right]$ is an edge of the lower convex hull of $U U^{\prime}$. The slopes of such edges are also given by the continued fraction expansion of the slope of $U U^{\prime}$. The connection between continued fractions and the convex hull of the lattice points located above or below a straight segment was already noticed by Klein in 1895 [3.

We do not provide further details due to lack of space, but the algorithm has been implemented in Lua and is available on the web1. It may be run as an ipelet in Ipe in order to draw the delaunay triangulation of any pattern described by a straight segment.

\subsection{Computation of the Voronoi Diagram}

The partial Voronoi diagram (Fig. 5,a), which is dual of $\mathcal{T}^{+}\left(U U^{\prime}\right)=\mathcal{F}\left(U U^{\prime}\right)$, is also computed by a simple recursive algorithm, because each vertex of the

${ }^{1}$ http://liris.cnrs.fr/tristan.roussillon/code/delaunay.lua 
diagram is the center of the circumcircle of a triangular facet of $\mathcal{F}\left(U U^{\prime}\right)$ and each edge of the diagram belongs to the bissector of an edge of a triangular facet of $\mathcal{F}\left(U U^{\prime}\right)$.

The algorithm may be coarsely described as follows: start with the point at infinity along the bissector of $\left[U U^{\prime}\right]$, then find $B=b^{-}\left(U U^{\prime}\right)$, the lower Bezout point of $U U^{\prime}$, using the extended Euclidean algorithm, link $\Omega$, the center of the circumcircle of $U B U^{\prime}$, to the previous vertex and split $U U^{\prime}$ into $U B$ and $B U^{\prime}$ in order to recursively apply this procedure on sub-patterns.

Let $B=(\beta, \alpha)$. Simple calculations lead to:

$$
\Omega=\left(\frac{a \cdot\left(\alpha^{2}+\beta^{2}\right)-\left(a^{2}+b^{2}\right) \cdot \alpha}{2}, \frac{\left(a^{2}+b^{2}\right) \cdot \beta-b \cdot\left(\alpha^{2}+\beta^{2}\right)}{2}\right)
$$

For instance, if $U^{\prime}=(5,3)$ and $B=(2,1), \Omega=\left(\frac{-19}{2}, \frac{43}{2}\right)$ ( $\Omega$ is the leftmost

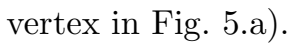

To sum up, the partial Voronoi diagram dual of $\mathcal{T}^{+}\left(U U^{\prime}\right)$ is a planar binary tree whose vertices have integer or half-integer coordinates and whose edges have a slope $-\frac{b_{k}}{a_{k}}$ such that $\frac{a_{k}}{b_{k}}$ is a fraction lying on the path going from the Stern-Brocot tree root to the fraction $\frac{a}{b}\left(\frac{3}{5}\right.$ in Fig. [5., b).

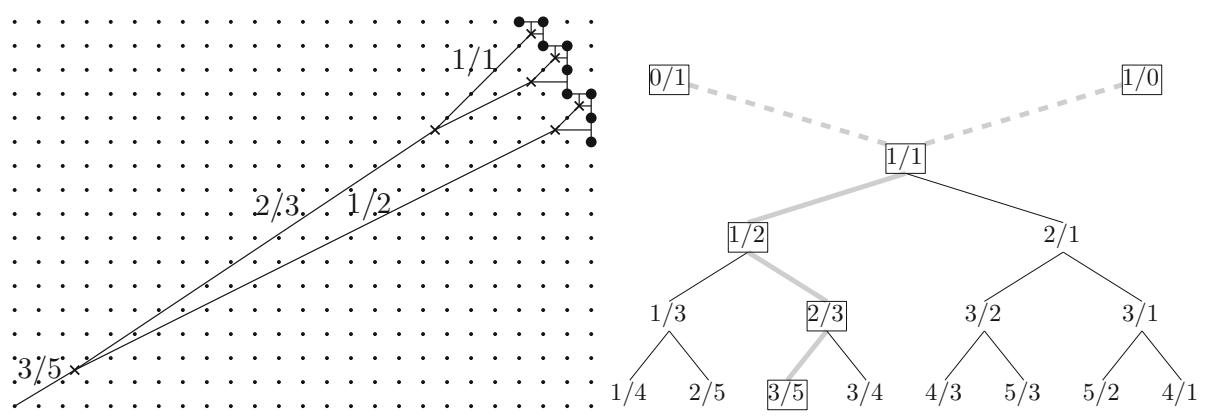

(a)

Fig. 5. The partial Voronoi diagram of a pattern of slope $\frac{3}{5}$ is depicted in (a). The figure is turned $90^{\circ}$ to the left so that the slopes of the edges are those lying on the path highlighted in (b), which goes from the Stern-Brocot tree root to the fraction $\frac{3}{5}$.

\section{Discussion}

The presented results may have several interesting applications in digital shape analysis. First of all, it may lead to new algorithms for computing the Delaunay triangulation of a digital shape, starting from a decomposition of its boundary into digital straight segments, for instance the one given by the minimum length polygon (see 8 for a recent algorithm). The input data would then be some $\Theta\left(n^{\frac{2}{3}}\right)$ instead of $n$ for digitization of smooth shapes. The Delaunay triangulation contains all the $\alpha$-shapes of its vertices [4, which is of great interest for 
shape reconstruction. The present work is also an essential step for enumerating the maximal digital circular arcs along digitizations of Euclidean shapes, since the center of these arcs are related to the Voronoi vertices. This enumeration would help for determining the multigrid convergence of several discrete curvature estimators, similarly to the number of maximal segments that was the key ingredients to determine the multigrid convergence of tangent estimators. Our results may also help in determining properties of the $\lambda$-medial axis $[2$ and its digital counterpart [1. Finally, a new class of geometric estimators from a set of points relies uniquely on the local shape of the Voronoi diagram 6]. Our results clearly avoid global Voronoi computation and may even give partial analytic quantities for that kind of applications.

\section{References}

1. Chaussard, J., Couprie, M., Talbot, H.: A discrete $\lambda$-medial axis. In: Brlek, S., Reutenauer, C., Provençal, X. (eds.) DGCI 2009. LNCS, vol. 5810, pp. 421-433. Springer, Heidelberg (2009)

2. Chazal, F., Lieutier, A.: The $\lambda$-medial axis. Graph. Models 67(4), 304-331 (2005)

3. Davenport, H.: The Higher Arithmetic. Cambridge University Press, Cambridge (1992)

4. Edelsbrunner, H., Kirkpatrick, D.G., Seidel, R.: On the Shape of a Set of Points in the Plane. IEEE Trans. on IT 29(4), 551-559 (1983)

5. Klette, R., Rosenfeld, A.: Digital straightness - a review. Discrete Applied Mathematics 139(1-3), 197-230 (2004)

6. Mérigot, Q., Ovsjanikov, M., Guibas, L.: Robust voronoi-based curvature and feature estimation. In: Proc. SIAM/ACM Joint Conference on Geometric and Physical Modeling (SPM 2009), pp. 1-12. ACM, New York (2009)

7. Preparata, F.P., Shamos, M.I.: Computational geometry: an introduction. Springer, Heidelberg (1985)

8. Provençal, X., Lachaud, J.O.: Two linear-time algorithms for computing the minimum length polygon of a digital contour. In: Brlek, S., Reutenauer, C., Provençal, X. (eds.) DGCI 2009. LNCS, vol. 5810, pp. 104-117. Springer, Heidelberg (2009)

9. Reveillès, J.P.: Géométrie discrète, calcul en nombres entiers et algorithmique. Thèse d'etat, Université Louis Pasteur, Strasbourg, France (1991) (in french)

10. Voss, K.: Discrete Images, Objects, and Functions in $\mathbb{Z}^{n}$. Springer, Heidelberg (1993) 